\title{
Gröbner bases over Tate algebras
}

\author{
Xavier Caruso \\ Université de Bordeaux, CNRS, \\ INRIA \\ Bordeaux, France \\ xavier.caruso@normalesup.org
}

\author{
Tristan Vaccon \\ Université de Limoges; CNRS, XLIM \\ UMR 7252 \\ Limoges, France \\ tristan.vaccon@unilim.fr
}

\author{
Thibaut Verron \\ Johannes Kepler University \\ Institute for Algebra \\ Linz, Austria \\ thibaut.verron@jku.at
}

\begin{abstract}
Tate algebras, introduced in [Ta71], are fundamental objects in the context of analytic geometry over the $p$-adics. Roughly speaking, they play the same role as polynomial algebras play in classical algebraic geometry. In the present article, we develop the formalism of Gröbner bases for Tate algebras. We prove an analogue of the Buchberger criterion in our framework and design a Buchbergerlike and a F4-like algorithm for computing Gröbner bases over Tate algebras. An implementation in SAGEMATH is also discussed.
\end{abstract}

\section{CCS CONCEPTS}

- Computing methodologies $\rightarrow$ Algebraic algorithms;

\section{KEYWORDS}

Algorithms, Power series, Tate algebra, Gröbner bases, F4 algorithm, $p$-adic precision

\section{ACM Reference Format:}

Xavier Caruso, Tristan Vaccon, and Thibaut Verron. 2019. Gröbner bases over Tate algebras. In International Symposium on Symbolic and Algebraic Computation (ISSAC '19), fuly 15-18, 2019, Beijing, China. ACM, New York, NY, USA, 9 pages. https://doi.org/10.1145/3326229.3326257

\section{INTRODUCTION}

In complex geometry, the concept of analytic functions is obviously a notion of first importance. They form a class of functions that exhibit strong rigidity properties as polynomials do but, at the same time, allow for many analytic constructions such as taking limits, integrals, etc. For this reason, they often appear as a bridge between algebra and analysis.

For many arithmetical applications, the completion $\mathbb{Q}_{p}$ of $\mathbb{Q}$ is often as relevant as $\mathbb{R}$ or $\mathbb{C}$. At the beginning of the 20th century, mathematicians realized that it would be quite interesting to develop the theory of $p$-adic analytic functions and eventually that of $p$-adic analytic geometry. However doing so is not an easy task owing to the totally disconnected topology on $\mathbb{Q}_{p}$.

The third author is supported by the Austrian FWF grant F5004.

Permission to make digital or hard copies of all or part of this work for personal or classroom use is granted without fee provided that copies are not made or distributed for profit or commercial advantage and that copies bear this notice and the full citation on the first page. Copyrights for components of this work owned by others than the author(s) must be honored. Abstracting with credit is permitted. To copy otherwise, or republish, to post on servers or to redistribute to lists, requires prior specific permission and/or a fee. Request permissions from permissions@acm.org.

ISSAC '19, July 15-18, 2019, Beijing, China

(C) 2019 Copyright held by the owner/author(s). Publication rights licensed to the As sociation for Computing Machinery.

ACM ISBN 978-1-4503-6084-5/19/07 . \$ \$15.00

https://doi.org/10.1145/3326229.3326257
In [Ta71], Tate proposed to replace the classical $p$-adic topology by some well-suited Grothendieck topology and came up with the notion of $p$-adic rigid variety. Basically, the construction of rigid varieties follow that of schemes in algebraic geometry. They are obtained by gluing pieces - the so-called affinoids - with respect to the aforementioned Grothendieck topology. As for affinoids, they are defined as the "spectrum" of quotients of some particular algebras, called Tate algebras. Thereby, Tate algebras play the same role in rigid geometry as polynomial algebras do in classical algebraic geometry.

From the purely algebraic point of view, Tate algebras have been widely studied and it has been demonstrated that they share some properties with polynomial algebras [BGR84]. However, as far as we know, the computational aspects of Tate algebras have not been developed yet. This contrasts with the polynomial setting, for which we have at our disposal the theory of Gröbner bases [Bu65, Co15], which has become over the years a research topic on its own. The aim of the present article is to extend the notion of Gröbner bases to Tate algebras.

Some difficulties need to be overcome. The most significant one is that elements in Tate algebras are, by nature, infinite convergent series and so they do not have a degree. This seems to be a serious obstruction since the degree is the most basic notion on which the classical theory of Gröbner bases is built. However, analyzing the definition of Tate algebras, we notice that a Tate series defines a sequence of polynomials (of growing degrees) by reduction modulo $p^{n}$ when $n$ varies. In order to take advantage of this observation, we introduce an order on the terms taking into account the $p$-adic valuation of the coefficients. This order is not well-founded as classical term orders are usually. However, we shall prove that it is topologically well-founded (in the sense that every decreasing sequence tends to 0 ) and that this weaker property is enough to guarantee the termination of our algorithms at finite precision.

Related works. Gröbner bases over rings - and in particular over $\mathbb{Z}$ and $\mathbb{Z} / n \mathbb{Z}$ - have also received some attention [AL94, KC09, NS01]. These developments are of course related to this article since quotients of Tate algebras are polynomial algebras over $\mathbb{Z} / p^{n} \mathbb{Z}$. The main difference between our point of view and that of loc. cit. appears in the choice of the term ordering; while, in the theory of Gröbner bases of rings, only the degree is considered, our setting forces us to include the valuation of the coefficients in the definition of the term ordering. It is the "price to pay" to be able to pass smoothly to the completion and catch inexact base rings as $\mathbb{Q}_{p}$.

The special term ordering we use comes from two different sources. The first one is the theory of tropical Gröbner bases by Chan and Maclagan [CM19] in which, for the first time, the valuation of the coefficients has been taken into account in the definition of the term ordering. Later on, Vaccon and his coauthors [ $\mathrm{Va}^{*}, \mathrm{Va} 15$, 
VY17, VVY18] observed that tropical orders are relevant for the computation of $p$-adic Gröbner bases as they improve substantially the numerical accuracy. The definition of our term order is the natural outcome of this observation. Our second source of inspiration is the theory of standard bases, which was designed originally to "compute" the singularities of algebraic varieties [Mo82, GR95] This theory introduces the notion of term order of local/mixed type, on which the term ordering we are using in the present article is modeled.

Structure of the article. In $\S 2$, we introduce Tate algebras and develop the theory of Gröbner bases over them. We prove in particular the existence of finite Gröbner bases and study their structure. $\S 3$ is devoted to algorithms. We first design a variant of the Buchberger algorithm that runs over Tate algebras. Several results towards its numerical stability are also presented. We then move to F4-like algorithms and show how they could be adapted to fit into the framework of Tate algebras. Finally, in §4, an implementation in SAgEMATH is briefly discussed.

Notation. The notation $\mathbb{N}$ will refer to the set of nonnegative integers (including 0 ). If $\mathfrak{A}$ is a ring, we will denote its group of invertible elements by $\mathfrak{A}^{\times}$. We fix a positive integer $n$. Let $X_{1}, \ldots, X_{n}$ be $n$ variables. We will use the short notation $\mathrm{X}$ for $\left(X_{1}, \ldots, X_{n}\right)$. Similarly for $\mathbf{i}=\left(i_{1}, \ldots, i_{n}\right) \in \mathbb{N}^{n}$, we shall write $\mathrm{X}^{\mathbf{i}}$ for $X_{1}^{i_{1}} \cdots X_{n}^{i_{n}}$.

\section{GRÖBNER BASES OVER TATE ALGEBRAS}

Throughout this article, we fix a field $K$ equipped with a discrete valuation val $: K \rightarrow \mathbb{Z} \sqcup\{+\infty\}$, normalized by $\operatorname{val}\left(K^{\times}\right)=\mathbb{Z}$. We shall always assume that $K$ is complete with respect to the distance defined by val. We let $K^{\circ}$ be the subring of $K$ consisting of elements of nonnegative valuation and $\pi$ be a uniformizer of $K$, that is an element of valuation 1 . We set $\bar{K}=K^{\circ} / \pi K^{\circ}$.

A typical example of $K$ as above is the field of $p$-adic numbers $\mathbb{Q}_{p}$ (equipped with the $p$-adic valuation). For this example, we have $K^{\circ}=\mathbb{Z}_{p}$ and $\bar{K}=\mathbb{F}_{p}$.

\subsection{Tate algebras}

We endow $\mathbb{R}^{n}$ with the usual scalar product $(x, y) \mapsto x \cdot y$.

Definition 2.1. Let $\mathbf{r}=\left(r_{1}, \ldots, r_{n}\right) \in \mathbb{Q}^{n}$. The Tate algebra $K\{\mathbf{X} ; \mathbf{r}\}$ is defined by:

$$
K\{\mathbf{X} ; \mathbf{r}\}:=\left\{\sum_{\mathbf{i} \in \mathbb{N}^{n}} a_{\mathbf{i}} \mathbf{X}^{\mathbf{i}} \text { s.t. } a_{\mathbf{i}} \in K \text { and } \operatorname{val}\left(a_{\mathbf{i}}\right)-\mathbf{r} \cdot \mathbf{i} \underset{|\mathbf{i}| \rightarrow+\infty}{\longrightarrow}+\infty\right\}
$$

The tuple $\mathbf{r}$ is called the convergence log-radii of the Tate algebra.

Elements of $K\{\mathbf{X} ; \mathbf{r}\}$ are the power series converging on the product of closed balls $B\left(0,|\pi|^{r_{1}}\right) \times \cdots \times B\left(0,|\pi|^{r_{n}}\right)$ where $|\cdot|$ is the absolute value on $K$ induced by val. When $\mathbf{r}=(0, \ldots, 0)$, we will simply write $K\{\mathbf{X}\}$ instead of $K\{\mathbf{X} ;(0, \ldots, 0)\}$.

Example 2.2. Let $K=\mathbb{Q}_{p}$. The series $f_{1}=\frac{1}{p}+X+p X^{2}+p^{2} X^{3}+$ $\ldots$ lies in $K\{X\}$. The series $f_{2}=1+X+X^{2}+X^{3}+\ldots$ does not lie in $K\{X\}$, because it does not converge when evaluated at 1 (for example). However, it does converge when evaluated at $x$ with $|x|<1$, so it lies in $K\{X ;(r)\}$ for all negative $r$.
The Tate algebra $K\{\mathbf{X} ; \mathbf{r}\}$ is equipped with the Gauss valuation $\operatorname{val}_{\mathbf{r}}: K\{\mathbf{X} ; \mathbf{r}\} \rightarrow \mathbb{Q} \sqcup\{+\infty\}$ defined as follows:

$$
\operatorname{val}_{\mathbf{r}}\left(\sum_{\mathbf{i} \in \mathbb{N}^{n}} a_{\mathbf{i}} X^{\mathbf{i}}\right)=\min _{\mathbf{i} \in \mathbb{N}^{n}}\left(\operatorname{val}\left(a_{\mathbf{i}}\right)-\mathbf{r} \cdot \mathbf{i}\right)
$$

We observe that the minimum is always reached thanks to the growth condition imposed in Definition 2.1. Moreover, the image of $v l_{r}$ is discrete. Geometrically, the Gauss valuation corresponds to the minimal valuation reached by the series on its domain of convergence (possibly after a finite extension of $K$ ).

Definition 2.3. The integral Tate algebra ring $K\{\mathrm{X} ; \mathbf{r}\}^{\circ}$ is defined as the subring of $K\{\mathbf{X} ; \mathbf{r}\}$ consisting of elements with nonnegative Gauss valuation.

Again we will use the notation $K\{\mathbf{X}\}^{\circ}$ for $K\{\mathbf{X} ;(0, \ldots, 0)\}^{\circ}$. When $\mathbf{r}=\left(r_{1}, \ldots, r_{n}\right) \in \mathbb{Z}^{n}$, observe that $K\{\mathbf{X} ; \mathbf{r}\}=K\left\{\pi^{r_{1}} X_{1}, \ldots, \pi^{r_{n}} X_{n}\right\}$ and similarly for $K\{\mathbf{X} ; \mathbf{r}\}^{\circ}$. The case $r \in \mathbb{Z}^{n}$ then reduces to $\mathbf{r}=0$ via a change of variables.

Example 2.4. With the notations of Example 2.2, $f_{1}$ does not lie in $K\{X\}^{\circ}$, but $f_{2}$ does lie in $K\{X ;(r)\}^{\circ}$ for all negative $r$.

Proposition 2.5. We have $K\{\mathbf{X} ; \mathbf{r}\}=K\{\mathbf{X} ; \mathbf{r}\}^{\circ}\left[\frac{1}{\pi}\right]$.

\subsection{About terms}

From now on, we fix a $\log$-radii $\mathbf{r} \in \mathbb{Q}^{n}$.

Monoids of terms. We first recall some basic definitions.

Definition 2.6. A monoid is a set equipped with a single associative binary operation, which has a neutral element.

An ideal of a monoid $M$ is a subset $I \subset M$ such that, for all $a \in M$ and $x \in I$, we have $a x \in I$.

We define the monoid of terms $T\{\mathbf{X} ; \mathbf{r}\}$ as the multiplicative monoid consisting of the elements $a \mathrm{X}^{\mathrm{i}}$ with $a \in K^{\times}$and $\mathbf{i} \in \mathbb{N}^{n}$. We let also $T\{\mathbf{X} ; \mathbf{r}\}^{\circ}$ be the submonoid of $T\{\mathrm{X} ; \mathbf{r}\}$ consisting of terms $a \mathbf{X}^{\mathbf{i}}$ for which $\operatorname{val}_{\mathbf{r}}\left(a \mathbf{X}^{\mathbf{i}}\right) \geq 0$. The multiplicative group $K^{\times}$(resp. $\left.\left(K^{\circ}\right)^{\times}\right)$embeds into $T\{\mathbf{X} ; \mathbf{r}\}\left(\right.$ resp. $\left.T\{\mathbf{X} ; \mathbf{r}\}^{\circ}\right)$. We set:

$$
\mathbb{T}\{\mathbf{X} ; \mathbf{r}\}=T\{\mathbf{X} ; \mathbf{r}\} / K^{\times} \text {and } \mathbb{T}\{\mathbf{X} ; \mathbf{r}\}^{\circ}=T\{\mathbf{X} ; \mathbf{r}\}^{\circ} /\left(K^{\circ}\right)^{\times} .
$$

The inclusion $T\{\mathbf{X} ; \mathbf{r}\}^{\circ} \subset T\{\mathbf{X} ; \mathbf{r}\}$ induces a canonical morphism (which is no longer injective) $\mathbb{T}\{\mathrm{X} ; \mathbf{r}\}^{\circ} \rightarrow \mathbb{T}\{\mathrm{X} ; \mathbf{r}\}$. The ideals of $\mathbb{T}\{\mathbf{X} ; \mathbf{r}\}$ (resp. of $\mathbb{T}\{\mathbf{X} ; \mathbf{r}\}^{\circ}$ ) are in bijective correspondence with the ideals of $T\{\mathbf{X} ; \mathbf{r}\}$ (resp. of $\left.T\{\mathbf{X} ; \mathbf{r}\}^{\circ}\right)$. Moreover, $\mathbb{T}\{\mathbf{X} ; \mathbf{r}\}$ and $\mathbb{T}\{\mathbf{X} ; \mathbf{r}\}^{\circ}$ do not contain non trivial invertible elements. In other words, the divisibility relation defines an order on $\mathbb{T}\{\mathbf{X} ; \mathbf{r}\}$ and $\mathbb{T}\{\mathbf{X} ; \mathbf{r}\}^{\circ}$. The following easy lemma elucidates the structure of $\mathbb{T}\{\mathbf{X} ; \mathbf{r}\}$ and $\mathbb{T}\{\mathbf{X} ; \mathbf{r}\}^{\circ}$

Lemma 2.7. (1) The mapping $\mathbb{T}\{\mathrm{X} ; \mathbf{r}\} \rightarrow \mathbb{N}^{n}, a \mathrm{X}^{\mathbf{i}} \mapsto \mathbf{i}$ is an isomorphism of monoids.

(2) The mapping $\mathbb{T}\{\mathbf{X} ; \mathbf{r}\}^{\circ} \rightarrow \mathbb{Q} \times \mathbb{N}^{n}$, aX $\mathbf{i} \mapsto\left(\operatorname{val}_{\mathbf{r}}\left(a \mathbf{X}^{\mathbf{i}}\right)\right.$, i) is an injective morphism of monoids; its image is included in $\frac{1}{D} \mathbb{N} \times \mathbb{N}^{n}$ where $D$ is a common denominator of the coordinates of $\mathbf{r}$.

(3) The natural morphism $\mathbb{T}\{\mathbf{X} ; \mathbf{r}\}^{\circ} \rightarrow \mathbb{T}\{\mathbf{X} ; \mathbf{r}\}$ corresponds to the projection onto the factor $\mathbb{N}^{n}$.

Proposition 2.8. Let I be an ideal of $\mathbb{T}\{\mathrm{X} ; \mathbf{r}\}$ (resp. of $\mathbb{T}\{\mathrm{X} ; \mathbf{r}\}^{\circ}$ ). Then there exists a unique subset $S$ of I having the two following properties: (1) $S$ generates I, and (2) every subset generating I contains $S$. Moreover $S$ is finite. 
Proof. The unicity is easy. Indeed if $S$ and $S^{\prime}$ satisfy (1) and (2), one must have $S \subset S^{\prime}$ and $S^{\prime} \subset S$, i.e. $S=S^{\prime}$. In order to prove the existence, we define $S$ as the set of minimal elements of $I$ for the divisibility relation. The fact that $S$ generates $I$ follows from the fact that divisibility is a well-founded order on $\mathbb{T}\{\mathbf{X} ; \mathbf{r}\}$ ( $c f$ Lemma 2.7). The point (2) is obvious.

It remains to prove that $S$ is finite. For this, we observe that any sequence with values in $\mathbb{N}$ necessarily has a nondecreasing subsequence. Extracting subsequences repeatedly, we find that the previous property also holds for sequences with values in $\mathbb{N}^{m}$ for any integer $m$. By Lemma 2.7, it also holds for sequences with values in $\mathbb{T}\{\mathbf{X} ; \mathbf{r}\}$ (resp. in $\left.\mathbb{T}\{\mathbf{X} ; \mathbf{r}\}^{\circ}\right)$. Therefore, if $S$ were not finite, we would be able to extract from $S$ a nondecreasing sequence. This contradicts the fact that $S$ is composed by minimal elements.

Definition 2.9. Let $I$ be an ideal of $\mathbb{T}\{\mathbf{X} ; \mathbf{r}\}\left(\right.$ resp. of $\left.\mathbb{T}\{\mathrm{X} ; \mathbf{r}\}^{\circ}\right)$. The subset $S$ of Proposition 2.8 is called the skeleton of $I$; it is denoted by $\operatorname{Skel}(I)$.

The skeleton of an ideal of $T\{\mathbf{X} ; \mathbf{r}\}$ (resp. of $T\{\mathbf{X} ; \mathbf{r}\}^{\circ}$ ) is defined as the skeleton of its image in $\mathbb{T}\{\mathbf{X} ; \mathbf{r}\}$ (resp. in $\mathbb{T}\{\mathbf{X} ; \mathbf{r}\}^{\circ}$ ); it is denoted by Skel(I).

In what follows, it will sometimes be convenient to work more generally with fractional ideals. By definition a fractional ideal of $\mathbb{T}\{\mathbf{X} ; \mathbf{r}\}^{\circ}$ is a subset of $\mathbb{T}\{\mathbf{X} ; \mathbf{r}\}$ which is stable by multiplication by elements in $\mathbb{T}\{\mathbf{X} ; \mathbf{r}\}^{\circ}$ and is contained in $\pi^{-N} \mathbb{T}\{\mathrm{X} ; \mathbf{r}\}^{\circ}$ for some $N \in \mathbb{N}$. The notion of skeleton can be extended to fractional ideals $I$ of $\mathbb{T}\{\mathrm{X} ; \mathbf{r}\}^{\circ}$. For such ideals, $\operatorname{Skel}(I)$ is a finite subset of $T\{\mathrm{X} ; \mathbf{r}\} /\left(K^{\circ}\right)^{\times}$ An interesting example of fractional ideal is:

$$
\mathbb{T}\{\mathbf{X} ; \mathbf{r}\}^{\geq v}=\left\{t \in \mathbb{T}\{\mathbf{X} ; \mathbf{r}\} \text { s.t. } \operatorname{val}_{\mathbf{r}}(t) \geq v\right\} .
$$

Remark 2.10. The effective computation of $\operatorname{Skel}(\mathbb{T}\{\mathbf{X} ; \mathbf{r}\} \geq v)$ is not an easy problem. It has been solved for $n=1$ in [CL14] using the theory of continued fractions. It would be interesting to generalize the results of loc. cit. to higher $n$.

Term order. We fix a monomial order $\leq_{\omega}$ on $\mathbb{N}^{n}$. We recall that this means that $\leq_{\omega}$ is a well-order which is compatible with the addition. Usual examples of monomial orders are lex, grevlex, etc.

Definition 2.11. We define a preorder $\leq$ on $T\{\mathrm{X} ; \mathbf{r}\}, T\{\mathrm{X} ; \mathbf{r}\}^{\circ}$ by:

$$
\begin{array}{lll}
a \mathbf{X}^{\mathbf{i}} \leq b \mathbf{X}^{\mathbf{j}} & \text { iff } & \operatorname{val}_{\mathbf{r}}\left(a \mathbf{X}^{\mathbf{i}}\right)>\operatorname{val}_{\mathbf{r}}\left(b \mathbf{X}^{\mathbf{j}}\right) \\
& \text { or } & \operatorname{val}_{\mathbf{r}}\left(a \mathbf{X}^{\mathbf{i}}\right)=\operatorname{val}_{\mathbf{r}}\left(b \mathbf{X}^{\mathbf{j}}\right) \text { and } \mathbf{i} \leq_{\omega} \mathbf{j} .
\end{array}
$$

Remark 2.12. The inequality sign is reversed in the first line: we require that $\operatorname{val}_{\mathbf{r}}\left(a \mathbf{X}^{\mathbf{i}}\right)>\operatorname{val}_{\mathbf{r}}\left(b \mathbf{X}^{\mathbf{j}}\right)$ and not $\operatorname{val}_{\mathbf{r}}\left(a \mathbf{X}^{\mathbf{i}}\right)<\operatorname{val}_{\mathbf{r}}\left(b \mathbf{X}^{\mathbf{j}}\right)$. This is not a typo and will be important in the sequel.

We underline that $\leq$ is not antisymmetric (and so not an order). More precisely, for $t_{1}, t_{2} \in T\{\mathbf{X} ; \mathbf{r}\}$, the fact that $t_{1} \leq t_{2}$ and $t_{2} \leq t_{1}$ is equivalent to the existence of $a \in\left(K^{\circ}\right)^{\times}$such that $t_{1}=a t_{2}$. As a consequence, $\leq$ induces an order on $\mathbb{T}\{\mathbf{X} ; \mathbf{r}\}^{\circ}$. On the contrary, we underline that $\leq$ does not factor through $\mathbb{T}\{X ; \mathbf{r}\}$.

Example 2.13. Let $K=\mathbb{Q}_{p}$ and consider $K\{X, Y\}$ with the lexicographical order. The preorder $\leq$ orders terms as follows:

$$
\begin{aligned}
\cdots>X Y^{2}>X Y & >X>\cdots>Y>1>\cdots \\
\cdots & >p X Y^{2}>\cdots>p>\cdots>p^{2} X Y^{2}>\cdots
\end{aligned}
$$

The terms $\mathbf{X}^{\mathbf{i}}$ and $-\mathbf{X}^{\mathbf{i}}$ are "equal" for $\leq$. So are $\mathbf{X}^{\mathbf{i}}$ and $(1+p) \mathbf{X}^{\mathbf{i}}$.
It is easily seen that the preorder $\leq$ is total. In turns out that it is not a well-order since the infinite sequence $\left(p^{n}\right)_{n \geq 0}$ is strictly decreasing. Nevertheless, we have:

Lemma 2.14. Let $\left(t_{j}\right)_{j \in \mathbb{N}}$ be a strictly decreasing sequence in $T\{\mathrm{X} ; \mathbf{r}\}$ (resp. in $\left.T\{\mathbf{X} ; \mathbf{r}\}^{\circ}\right)$. Then $\lim _{j \rightarrow \infty} \operatorname{val}_{\mathbf{r}}\left(t_{j}\right)=+\infty$.

Proof. From the definition of $\leq$, it follows that the sequence $\left(\operatorname{val}_{\mathbf{r}}\left(t_{j}\right)\right)_{j \in \mathbb{N}}$ is nondecreasing. Moreover it takes its values in $\frac{1}{D} \mathbb{N}$ for some positive integer $D$. Finally, the fact that $\leq_{\omega}$ is a well-order implies that for each fixed $v \in \frac{1}{D} \mathbb{N}$, there is only a finite number of indices $j$ for which $\operatorname{val}_{\mathbf{r}}\left(t_{j}\right)=v$. Combining these inputs, we find that $\operatorname{val}_{\mathbf{r}}\left(t_{j}\right)$ must tend to $+\infty$.

We notice that if $\mathbf{i} \neq \mathbf{j}$, the terms $a_{\mathbf{i}} X^{\mathbf{i}}$ and $a_{\mathbf{j}} X^{\mathbf{j}}$ are never "equal" for $\leq$. Therefore, any nonzero series $f=\sum_{\mathbf{i} \in \mathbb{N}^{n}} a_{\mathbf{i}} X^{\mathbf{i}} \in K\{\mathbf{X} ; \mathbf{r}\}$ has a unique leading term. We denote it $L T(f)$.

Example 2.15. With the notations of Example 2.13, the leading term of $g_{2}=X Y+p+p^{2} X Y$ is $L T\left(g_{2}\right)=\left(1+p^{2}\right) X Y$.

\subsection{Gröbner bases}

Definition 2.16. Given an ideal $J$ of $K\{\mathrm{X} ; \mathbf{r}\}\left(\right.$ resp. of $K\{\mathrm{X} ; \mathbf{r}\}^{\circ}$ ), we denote by $L T(J)$ the subset of $T\{\mathbf{X} ; \mathbf{r}\}$ (resp. of $\left.T\{\mathbf{X} ; \mathbf{r}\}^{\circ}\right)$ consisting of elements of the form $L T(f)$ with $f \in J, f \neq 0$.

We check immediately that $L T(J)$ is an ideal of the monoid $T\{\mathrm{X} ; \mathbf{r}\}$ (resp. of $T\{\mathbf{X} ; \mathbf{r}\}^{\circ}$ ).

Definition 2.17. Let $J$ be an ideal of $K\{\mathbf{X} ; \mathbf{r}\}$ (resp. of $K\{\mathbf{X} ; \mathbf{r}\}^{\circ}$ ). A family $\left(g_{1}, \ldots, g_{s}\right) \in J^{s}$ is a Gröbner basis (in short, GB) of $J$ if $L T(J)$ is generated by the $L T\left(g_{i}\right)^{\prime}$ s in $T\{\mathrm{X} ; \mathbf{r}\}$ (resp. in $\left.T\{\mathrm{X} ; \mathbf{r}\}^{\circ}\right)$.

Proposition 2.18. Let $G=\left(g_{1}, \ldots, g_{s}\right)$ be a $G B$ of an ideal $J$ of $K\{\mathrm{X} ; \mathbf{r}\}\left(\right.$ resp. of $\left.K\{\mathbf{X} ; \mathbf{r}\}^{\circ}\right)$. Then $G$ generates $J$.

Proof. Let $f \in J$. We define inductively a sequence $\left(f_{j}\right)_{j \in \mathbb{N}}$ as follows. Let $f_{0}=f$. Given $j$, we write $L T\left(f_{j}\right)=a_{j} \mathrm{X}^{\mathbf{i}_{j}} L T\left(g_{i_{j}}\right)$ and define $f_{j+1}=f_{j}-a_{j} \mathbf{X}^{\mathbf{i}_{j}} g_{i_{j}}$. Then $L T\left(f_{j+1}\right)<L T\left(f_{j}\right)$. By Lemma 2.14, $\operatorname{val}_{\mathbf{r}}\left(L T\left(f_{j}\right)\right)=\operatorname{val}_{\mathbf{r}}\left(f_{j}\right)$ goes to infinity when $j$ goes to infinity. Therefore we can then write $f=\sum_{j} a_{j} \mathbf{X}^{\mathbf{i}_{j}} g_{i_{j}}$ as a converging series. By regrouping terms, we get $f \in\left\langle g_{1}, \ldots, g_{s}\right\rangle$.

Proposition 2.8 gives a lot of information about the ideal $L T(J)$ (where $J$ is an ideal of $K\{\mathbf{X} ; \mathbf{r}\}$ or $K\{\mathbf{X} ; \mathbf{r}\}^{\circ}$ ). These results have interesting consequences on Gröbner bases.

TheOrem 2.19. Any ideal of $K\{\mathbf{X} ; \mathbf{r}\}$ or $K\{\mathbf{X} ; \mathbf{r}\}^{\circ}$ has a finite $G B$.

Proof. Let $t_{1}, \ldots, t_{s}$ be the elements of $\operatorname{Skel}(L T(J))$. For all $i$, let $g_{i} \in J$ be such that $L T\left(g_{i}\right)=t_{i}$ in $\mathbb{T}\{\mathbf{X} ; \mathbf{r}\}$ (resp. in $\left.\mathbb{T}\{\mathbf{X} ; \mathbf{r}\}^{\circ}\right)$. Then $\left(g_{1}, \ldots, g_{s}\right)$ is a GB of $J$.

Remark 2.20. Combining the previous theorem with Proposition 2.18, we obtain that any ideal of $K\{\mathbf{X} ; \mathbf{r}\}$ (resp. of $K\{\mathbf{X} ; \mathbf{r}\}^{\circ}$ ) is finitely generated. In other words, we have proved that the rings $K\{\mathrm{X} ; \mathbf{r}\}$ and $K\{\mathbf{X} ; \mathbf{r}\}^{\circ}$ are Noetherian (which was of course already known for a long time).

Another important consequence of Proposition 2.8 is the notion of minimal GB that we discuss now. 
Definition 2.21. Let $J$ be an ideal of $K\{\mathbf{X} ; \mathbf{r}\}$ (resp. of $K\{\mathbf{X} ; \mathbf{r}\}^{\circ}$ ). A GB $G=\left(g_{1}, \ldots, g_{s}\right)$ is minimal if the images in $\mathbb{T}\{\mathbf{X} ; \mathbf{r}\}$ (resp. in $\left.\mathbb{T}\{\mathbf{X} ; \mathbf{r}\}^{\circ}\right)$ of the $L T\left(g_{i}\right)^{\prime}$ 's are exactly the elements of Skel $(L T(J))$, with no repetition.

A direct consequence of the definition is that two minimal GB of a given ideal $J$ have the same cardinality, namely the cardinality of $\operatorname{Skel}(L T(J))$.

Theorem 2.22. Let $J$ be an ideal of $K\{\mathbf{X} ; \mathbf{r}\}$ (resp. of $\left.K\{\mathbf{X} ; \mathbf{r}\}^{\circ}\right)$. Let $G$ be a $G B$ of J. Then, there exists a subset $G^{\prime} \subset G$ which is a minimal $G B$ of $J$.

Proof. By definition, $L T(G)$ is a generating set of $L T(J)$. From Proposition 2.8, we deduce that $\operatorname{Skel}(L T(J)) \subset G$. For each term $t \in \operatorname{Skel}(L T(J))$, let us choose $g_{t} \in G$ such that $L T\left(g_{t}\right)=t$. The subset $G^{\prime}$ of $G$ consisting of such $g_{t}$ 's is then a minimal GB of $J$.

\subsection{Comparison results}

So far, we have defined a notion of GB for ideals of $K\{\mathrm{X} ; \mathbf{r}\}$ and $K\{\mathrm{X} ; \mathbf{r}\}^{\circ}$. The aim of this subsection is to compare them.

Proposition 2.23. Let $I$ be an ideal of $K\{\mathrm{X} ; \mathbf{r}\}^{\circ}$ and let $G$ be $a$ $G B$ of $I$. Then $G$ is a $G B$ of the ideal $J=I\left[\frac{1}{\pi}\right]$ of $K\{\mathbf{X} ; \mathbf{r}\}$.

Proof. It follows from the fact $L T(J)=L T(I)\left[\frac{1}{\pi}\right]$.

Remark 2.24. Note that minimality of GB is not preserved when passing from $K\{\mathbf{X} ; \mathbf{r}\}^{\circ}$ to $K\{\mathbf{X} ; \mathbf{r}\}$. For example, $G=(p, X)$ is a minimal GB of the ideal $I=(p, X)$ of $K^{\circ}\{X\}$. However it is not a minimal GB of $J=I\left[\frac{1}{\pi}\right]=K\{X\}$ since $p$ divides $X$ in this ring.

Going in the other direction (i.e. from $K\{\mathbf{X} ; \mathbf{r}\}$ to $K\{\mathbf{X} ; \mathbf{r}\}^{\circ}$ ) is more subtle. First of all, we remark that, if we start with an ideal $J$ of $K\{\mathrm{X} ; \mathbf{r}\}$, there exist many ideals $I$ of $K\{\mathrm{X} ; \mathbf{r}\}^{\circ}$ with the property that $I\left[\frac{1}{\pi}\right]=J$. However, the set of such ideals $I$ has a unique maximal element (for the inclusion); it is the ideal $J^{\circ}=J \cap K\{\mathrm{X} ; \mathbf{r}\}^{\circ}$. This special ideal $J^{\circ}$ can also be caracterized by the fact that it is $\pi$-saturated.

Proposition 2.25. Let $J$ be an ideal of $K\{\mathbf{X} ; \mathbf{r}\}$ and let $G=\left(g_{1}, \ldots, g_{s}\right)$ be a $G B$ (resp. a minimal GB) of J. We assume that $\operatorname{val}_{\mathbf{r}}\left(g_{i}\right)=0$ for all $i$. Then $G$ is a $G B$ (resp. a minimal $G B)$ of $J^{\circ}$.

Proof. Let $G$ be a GB of $J$. Let $t \in L T\left(J^{\circ}\right)$. Then $t$ is a multiple of one of the $L T\left(g_{i}\right)^{\prime}$ 's in $T\{\mathbf{X} ; \mathbf{r}\}$. Since $v^{2} \mathbf{r}_{\mathbf{r}}\left(g_{i}\right)=0$, we deduce that $L T\left(g_{i}\right)$ divides $t$ in $T\{\mathbf{X} ; \mathbf{r}\}^{\circ}$ as well. Consequently $G$ is a GB of $J^{\circ}$. The fact that minimality is preserved is easy.

When $\mathbf{r} \in \mathbb{Z}^{n}$, it is easy to build a GB of $J$ satisfying the assumption of Proposition 2.25 from any GB of $J$. Indeed if $\left(g_{1}, \ldots, g_{s}\right)$ is a GB of $J$ then $\operatorname{val}_{\mathbf{r}}\left(g_{i}\right)$ is an integer for all $i$ and the family $\left(\pi^{-\operatorname{val}_{\mathbf{r}}\left(g_{1}\right)} g_{1}, \ldots, \pi^{-\operatorname{val}_{\mathbf{r}}\left(g_{s}\right)} g_{s}\right)$ is a GB of $J$. On the contrary, when $\mathbf{r} \notin \mathbb{Z}^{n}$, the problem is more complicated (see example above).

Example 2.26. Choose $n=1$ and $\mathbf{r}=\left(\frac{1}{2}\right)$ and let $J$ be ideal of $K\{X\}$ generated by $X$. The ideal $J^{\circ}$ is then generated by $g_{1}=\pi X$ and $g_{2}=\pi X^{2}$. More precisely, one checks that $\left(g_{1}, g_{2}\right)$ is a minimal GB of $J^{\circ}$. In particular, we observe that the cardinality of a minimal GB of $J$ does not agree with that of a minimal GB of $J^{\circ}$.

For a general $\mathbf{r} \in \mathbb{Q}^{n}$, Proposition 2.25 can be generalized as follows.
Proposition 2.27. Let $J$ be an ideal of $K\{\mathbf{X} ; \mathbf{r}\}$ and let $G=\left(g_{1}, \ldots, g_{s}\right)$ be a GB of $J$. Then a $G B$ of $J^{\circ}$ is $\left(t_{i, j} g_{i}\right)$ 's where, for each fixed $i$, the $t_{i, j}$ 's enumerate the elements of $\operatorname{Skel}\left(T\{X ; \mathbf{r}\} \geq-\operatorname{val}_{\mathbf{r}}\left(g_{i}\right)\right)(\operatorname{cf} E q .(1))$.

Proof. From val $\mathbf{r}\left(t_{i, j}\right) \geq-\operatorname{val}_{\mathbf{r}}\left(g_{i}\right)$, we deduce that $t_{i, j} g_{i}$ lies in $K\{\mathrm{X} ; \mathbf{r}\}^{\circ}$ and hence in $J^{\circ}$. It remains to prove that the $L T\left(t_{i, j} g_{i}\right)^{\text {'s }}$ generate $L T\left(J^{\circ}\right)$. Let $\tau \in L T\left(J^{\circ}\right)$. Since $G$ is a GB of $J$, there exists an index $i$ such that $L T\left(g_{i}\right)$ divides $t$. Write $\tau=t L T(g)$. We have $\operatorname{val}_{\mathbf{r}}(\tau)=\operatorname{val}_{\mathbf{r}}(t)-\operatorname{val}_{\mathbf{r}}(g)$. From $\tau \in K\{\mathbf{X} ; \mathbf{r}\}^{\circ}$, we deduce that $\operatorname{val}_{\mathbf{r}}(\tau) \geq 0$ and then $\operatorname{val}_{\mathbf{r}}(t) \geq-\operatorname{val}_{r}\left(g_{i}\right)$. Consequently, $t$ is divisible by some $t_{i, j} \in \operatorname{Skel}\left(T\{\mathbf{X} ; \mathbf{r}\}^{\geq-\operatorname{val}_{\mathbf{r}}\left(g_{i}\right)}\right)$. We deduce that $\tau$ is divisible by $t_{i, j} L T\left(g_{i}\right)=L T\left(t_{i, j} g_{i}\right)$, as wanted.

Reduction in the residue field. When $\mathbf{r}=(0, \ldots, 0)$, the quotient $K\{\mathrm{X}\}^{\circ} / \pi K\{\mathrm{X}\}^{\circ}$ is isomorphic to the polynomial algebra $\bar{K}[\mathrm{X}]$, on which we have a well-defined notion of Gröbner bases.

Proposition 2.28. Let $J$ be an ideal of $K\{\mathrm{X}\}$. Set $J^{\circ}=J \cap K\{\mathrm{X}\}^{\circ}$ and let $\bar{J}^{\circ}$ be the image of $J^{\circ}$ in $\bar{K}[\mathrm{X}]$. Let $g_{1}, \ldots, g_{s}$ in $J$ be such that $\operatorname{val}_{0}\left(g_{i}\right)=0$ and let $\bar{g}_{1}, \ldots, \bar{g}_{s}$ be their images in $\bar{J}^{\circ}$. Then the following assertions are equivalent:

(1) $\left(g_{1}, \ldots, g_{s}\right)$ is a $G B$ of $J$;

(2) $\left(g_{1}, \ldots, g_{s}\right)$ is a $G B$ of $J^{\circ}$;

(3) $\left(\bar{g}_{1}, \ldots, \bar{g}_{s}\right)$ is a $G B$ of $\bar{J}^{\circ}$.

Proof. The equivalence between (1) and (2) has been already proved. We now prove that (2) implies (3). Let $\bar{f} \in \bar{J}^{\circ}$ and let $f \in J^{\circ}$ be a lift of $\bar{f}$. We can write $L T(f)=a X^{\mathbf{i}} L T\left(g_{i}\right)$ for some $a$, i and $i$. Then $L T(\bar{f})=\bar{a} X^{\mathrm{i}} L T\left(\bar{g}_{i}\right)$. Therefore the $L T\left(\bar{g}_{i}\right)$ 's generate $L T\left(\bar{J}^{\circ}\right)$. We prove finally that (3) implies (2). Let $f \in J^{\circ}$. Set $h=\pi^{-\operatorname{val}_{0}(f)} f$. Clearly $h \in J$ and $h \in K\{\mathbf{X}\}^{\circ}$. Thus $h \in J^{\circ}$. By (3), we can write $L T(\bar{h})=\bar{a} X^{\mathbf{i}} L T\left(\bar{g}_{i}\right)$ for $\bar{a} \in \bar{K}$ and $\mathbf{i} \in \mathbb{N}^{n}$. We write $L T(h)=h_{0} X^{H}$ with $h_{0} \in\left(K^{\circ}\right)^{\times}$and similarly, $L T\left(g_{i}\right)=b_{0} X^{F}$ with $b_{0} \in\left(K^{\circ}\right)^{\times}$. Then $X^{F}$ divides $X^{H}$. Let $L$ be such that $X^{H}=X^{F} \cdot X^{L}$. Then $L T(h)=h_{0} b_{0}^{-1} X^{L} L T\left(g_{i}\right)$ with $\frac{h_{0}}{b_{0}} \in K^{\circ}$.

\section{ALGORITHMS}

\subsection{Division and membership test}

Not surprisingly, Gröbner bases can be used to test membership in ideals. Before going further in this direction, we need to adapt the division algorithm to our setting. We will need two variants depending on where we are looking for the quotients.

Proposition 3.1. Let $f, h_{1}, \ldots, h_{m} \in K\{\mathrm{X} ; \mathbf{r}\}$. Then, there exist $q_{1}, \ldots, q_{m} \in K\{\mathbf{X} ; \mathbf{r}\}$ (resp. $\left.q_{1}, \ldots, q_{m} \in K\{\mathbf{X} ; \mathbf{r}\}^{\circ}\right)$ and $r \in$ $K\{\mathrm{X} ; \mathbf{r}\}$ such that:

(1) $f=q_{1} h_{1}+\cdots+q_{m} h_{m}+r$,

(2) for all $i$ and all terms $t$ of $r, L T\left(h_{i}\right) \nmid t$ in $T\{\mathbf{X} ; \mathbf{r}\}$ (resp. in $\left.T\{\mathbf{X} ; \mathbf{r}\}^{\circ}\right)$,

(3) for all terms $t_{i}$ of $q_{i}$, we have $L T\left(t_{i} h_{i}\right) \leq L T(f)$.

Proof. We only give the proof of $K\{\mathrm{X} ; \mathbf{r}\}$, the case of $K\{\mathrm{X} ; \mathbf{r}\}^{\circ}$ being totally similar. We will construct by induction sequences $\left(f_{j}\right)_{j \geq 0},\left(q_{i, j}\right)_{j \geq 0}(1 \leq i \leq m)$ and $\left(r_{j}\right)_{j \geq 0}$ such that:

$$
f=f_{j}+q_{1, j} h_{1}+\cdots+q_{m, j} h_{j}+r_{j}
$$

We set $f_{0}=f, r_{0}=0$ and $q_{1,0}=\cdots=q_{m, 0}=0$. If $L T\left(f_{j}\right)$ is divisible by some $L T\left(h_{i_{j}}\right)$, we set $f_{j+1}=f_{j}-\frac{L T\left(f_{j}\right)}{L T\left(h_{i}\right)} h_{i}$ and $q_{i_{j}, j+1}=$ 
$q_{i j, j}+\frac{L T\left(f_{j}\right)}{L T\left(h_{i}\right)}$, and leave unchanged $r$ and the others $q_{i}$ 's. Otherwise, we set $f_{j+1}=f_{j}-L T\left(f_{j}\right)$ and $r_{j+1}=r_{j}+L T\left(f_{j}\right)$.

If follows from the construction that $L T\left(f_{j+1}\right)<L T\left(f_{j}\right)$ for all $j$. By Lemma 2.14, $\lim _{j \rightarrow \infty} \operatorname{val}_{\mathbf{r}}\left(f_{j}\right)=+\infty$, i.e. $\left(f_{j}\right)_{j \geq 0}$ converges to 0 in $K\{\mathbf{X} ; \mathbf{r}\}$. Besides, $\operatorname{val}_{\mathbf{r}}\left(\frac{L T\left(f_{j}\right)}{L T\left(h_{i}\right)}\right)$ tends to infinity as well, so that the sequences $\left(q_{i, j}\right)_{j \geq 0}$ all converge. Combining this with Eq. (2), we find that $\left(r_{j}\right)_{j \geq 0}$ also converges. The elements $q_{i}=\lim _{j \rightarrow \infty} q_{i, j}$ and $r=\lim _{j \rightarrow \infty} r_{j}$ satisfy the requirements of the proposition.

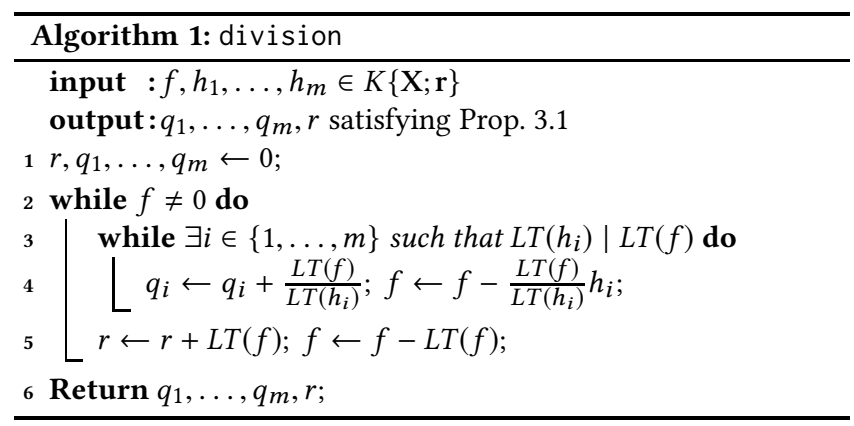

Algorithm 1 above summarizes the proof of Proposition 3.1. In general, it does not terminate, keeping computing more and more accurate approximations of the $q_{i}$ 's and $r$. However, in the common case where the coefficients of the input series are all known up to finite precision, i.e. modulo $\pi^{N}$ for some $N$, Algorithm 1 does terminate.

Remark 3.2. When working at finite precision, it is more intelligent, instead of computing the quotient $\frac{L T(f)}{L T\left(h_{i}\right)}$ (which would possibly lead to losses of precision), to choose an exact term $t$ such that the equality $L T(f)=t \cdot L T\left(h_{i}\right)$ holds at the working precision, and use it on lines 4 and 5. Doing so, we limit the losses of precision.

In general, the conditions of Proposition 3.1 are not enough to determine uniquely the $q_{i}$ 's and $r$. However, Proposition 3.3 below provides a weak unicity result when $\left(h_{1}, \ldots, h_{m}\right)$ is a Gröbner bases, which can be used to test membership.

Proposition 3.3. Let $J$ be an ideal of $K\{\mathrm{X} ; \mathbf{r}\}$ (resp. of $\left.K\{\mathrm{X} ; \mathbf{r}\}^{\circ}\right)$ and let $\left(g_{1}, \ldots, g_{s}\right)$ be a $G B$ of $J$. Let $f \in K\{\mathrm{X} ; \mathbf{r}\}$. We assume that we are given a decomposition $f=q_{1} g_{1}+\cdots+q_{s} g_{s}+r$ satisfying the requirements of Proposition 3.1. Then $r=0$ if and only if $f \in J$.

Proof. The "only if" is clear. Conversely, assume by contradiction that $f \in J$ and $r \neq 0$. Then $L T(r)$ makes sense. From the conditions of Proposition 3.1, we deduce that, for all $i, L T(r)$ is not divisible by $L T\left(g_{i}\right)$ Hence $L T(r) \notin L T(J)$, contradicting $r \in J$.

Remark 3.4. In the integral Tate algebra setting, it is not true that the remainder in the division by Gröbner bases is unique. For example, the division in $K\{\mathrm{X}\}^{\circ}$ of $f=1+p$ by $h=p$ can be written either $f=0 \times h+(1+p)$ or $f=1 \times h+1$. This is a general limitation of Gröbner bases over rings, even in the polynomial case [AL94].

\subsection{Buchberger's algorithm}

In this subsection, we adapt Buchberger's algorithm to fit into the framework of Tate algebras. The adaptation is more or less straightforward except on two points. The first one is related to finite precision, as already encountered previously. The second point is of different nature; it is related to the fact that, when the log-radii are not integers, the crucial notion of S-polynomials is not welldefined as the monoid $T\{\mathbf{X} ; \mathbf{r}\}^{\circ}$ does not admit gcd's. In what follows, we will give satisfying answers to these issues.

Buchberger's criterion. To begin with, we assume $\mathbf{r}=(0, \ldots, 0)$. In what follows, in order to simplify notations, we will write val instead of $\operatorname{val}_{(0, \ldots, 0)}$. Under this hypothesis, the monoid of terms $T\{\mathbf{X}\}$ admits gcd's and lcm's. Concretely we define:

$$
\begin{aligned}
& \operatorname{gcd}\left(a \mathbf{X}^{\mathbf{i}}, b \mathbf{X}^{\mathbf{j}}\right)=\pi^{\min (\operatorname{val}(a), \operatorname{val}(b))} X^{\inf (\mathbf{i}, \mathbf{j})}, \\
& \operatorname{lcm}\left(a \mathbf{X}^{\mathbf{i}}, b \mathbf{X}^{\mathbf{j}}\right)=\pi^{\max (\operatorname{val}(a), \operatorname{val}(b))} X^{\sup (\mathbf{i}, \mathbf{j})}
\end{aligned}
$$

where the inf and the sup over $\mathbb{N}^{n}$ are taken coordinate by coordinate. If $t_{1}$ and $t_{2}$ are two terms, the valuation of $\operatorname{gcd}\left(t_{1}, t_{2}\right)$ (resp. of $\left.\operatorname{lcm}\left(t_{1}, t_{2}\right)\right)$ is the minimum (resp. the maximum) of $\operatorname{val}\left(t_{1}\right)$ and $\operatorname{val}\left(t_{2}\right)$.

Definition 3.5. For $f, g$ in $K\{\mathrm{X}\}$, we define:

$$
S(f, g)=\frac{L T(g)}{\operatorname{gcd}(L T(f), L T(g))} f-\frac{L T(f)}{\operatorname{gcd}(L T(f), L T(g))} g .
$$

We have the following generalization of [Bu65, Sec. 2.10, Prop. 5]:

LEMmA 3.6. Let $h_{1}, \ldots, h_{m} \in K\{\mathbf{X}\}$ and $t_{1}, \ldots, t_{m} \in T\{\mathbf{X}\}$. We assume that the $L T\left(t_{i} h_{i}\right)$ 's all have the same image in $T\{\mathrm{X}\} /\left(K^{\circ}\right)^{\times}$ and that $L T\left(\sum_{i=1}^{m} t_{i} h_{i}\right)<L T\left(t_{i} h_{i}\right)$. Then

$$
\sum_{i=1}^{m} t_{i} h_{i}=\sum_{i=1}^{m-1} t_{i}^{\prime} \cdot S\left(h_{i}, h_{i+1}\right)+t_{m}^{\prime} \cdot h_{m}
$$

for some $t_{1}^{\prime}, \ldots, t_{m}^{\prime} \in T\{\mathrm{X}\}$ such that $\operatorname{val}\left(t_{m}^{\prime} h_{m}\right)>\operatorname{val}\left(t_{1} h_{1}\right)$ and $\operatorname{val}\left(t_{i}^{\prime}\right)+\max \left(\operatorname{val}\left(h_{i}\right), \operatorname{val}\left(h_{i+1}\right)\right) \geq \operatorname{val}\left(t_{1} h_{1}\right)$ for $i \in\{1, \ldots, m-1\}$.

Proof. By assumption, there exist $\mathbf{i} \in \mathbb{N}^{n}$ and $d_{1}, \ldots, d_{n} \in K^{\times}$ such that $L T\left(t_{i} h_{i}\right)=d_{i} \mathrm{X}^{\mathbf{i}}$ for all $i \in\{1, \ldots, m\}$. Moreover all the $d_{i}$ 's have the same valuation, say $\mu$. Then $\operatorname{val}\left(\sum_{i} d_{i}\right)>\mu$. We define $p_{i}=\frac{t_{i} h_{i}}{d_{i}}$, so that $t_{i} h_{i}=d_{i} p_{i}$. Then

$$
\begin{aligned}
\sum_{i} t_{i} h_{i}= & d_{1}\left(p_{1}-p_{2}\right)+\left(d_{1}+d_{2}\right)\left(p_{2}-p_{3}\right)+\cdots+ \\
& \left(d_{1}+\cdots+d_{m-1}\right)\left(p_{m-1}-p_{m}\right)+\left(d_{1}+\cdots+d_{m}\right) p_{m} .
\end{aligned}
$$

Observing that $p_{i}-p_{i+1}=t_{i}^{\prime} \cdot S\left(h_{i}, h_{i+1}\right)$ with $t_{i}^{\prime} \in T\{\mathbf{X}\}$, we get the lemma.

Theorem 3.7. Let $h_{1}, \ldots, h_{s}$ be elements of $K\{\mathrm{X}\}$ (resp. of $\left.K\{\mathrm{X}\}^{\circ}\right)$ and let I be the ideal of $K\{\mathrm{X}\}$ (resp. of $\left.K\{\mathrm{X}\}^{\circ}\right)$ generated by the $h_{i}$ 's. Then $\left(h_{1}, \ldots, h_{s}\right)$ is a GB of I if and only if all $S\left(h_{i}, h_{j}\right), i \neq j$, reduce to zero after division by $\left(h_{1}, \ldots, h_{s}\right)$ using Algorithm 1.

Proof. The "only if" part follows from Proposition 3.3. We prove the "if" part. Let us assume by contradiction that there exists some $f \in I$ such that $L T(f) \notin\left\langle L T\left(h_{i}\right)\right\rangle$. We can write $f=\sum_{i} q_{i} h_{i}$ with $q_{i} \in K\{\mathbf{X}\}$ (resp. $q_{i} \in K\{\mathbf{X}\}^{\circ}$ ). Let $t$ be maximal among the $L T\left(q_{i} h_{i}\right)$ 's. We have $L T(f)<t$ because of the hypothesis that 
$L T(f) \notin\left\langle L T\left(h_{i}\right)\right\rangle$. We can moreover assume that the decomposition $f=\sum_{i} q_{i} h_{i}$ is chosen in such a way that $t$ is minimal.

Let $J$ be the set of indices $i$ for which $L T\left(q_{i} h_{i}\right)=a \cdot t$ for some $a \in\left(K^{\circ}\right)^{\times}$. Set $t_{i}=L T\left(q_{i}\right)$ for $i \in J$ and define $h=\sum_{i \in J} t_{i} h_{i}$; we have $L T(h)<t$. Applying Lemma 3.6, we find $j_{0} \in J$ and terms $t^{\prime}, t_{j, k}^{\prime}($ for $j, k \in J)$ such that $h=\sum_{j, k \in J} t_{j, k}^{\prime} S\left(h_{j}, h_{k}\right)+t^{\prime} h_{j_{0}}$ and $\operatorname{val}\left(t^{\prime} h_{j_{0}}\right)>\operatorname{val}(h), \operatorname{val}\left(t_{j, k}^{\prime}\right)+\min \left(\operatorname{val}\left(h_{j}\right), \operatorname{val}\left(h_{j}\right)\right) \geq \operatorname{val}(h)$ Applying Proposition 3.1 with the S-polynomials, and using the fact that the leading terms of the summands in an S-polynomial cancel out, we get $b_{1}, \ldots, b_{m} \in K\{\mathbf{X}\}$ such that $h=\sum_{i=1}^{m} b_{i} h_{i}$ and $L T\left(b_{i} h_{i}\right)<t$ for all $i$. Therefore, we find that $f$ can be written as $f=\sum_{i \in \mathbf{i}} q_{i}^{\prime} h_{i}$ with $q_{1}^{\prime}, \ldots, q_{m}^{\prime} \in K\{\mathbf{X}\}$ and $L T\left(q_{i}^{\prime} h_{i}\right)<t$ for all $i$. This contradicts the minimality of $t$.

Buchberger's algorithm. After Theorem 3.7, it is easy to design a Buchberger type algorithm for computing GB over $K\{\mathrm{X}\}$ and $K\{\mathrm{X}\}^{\circ}$. It is Algorithm 2 below.

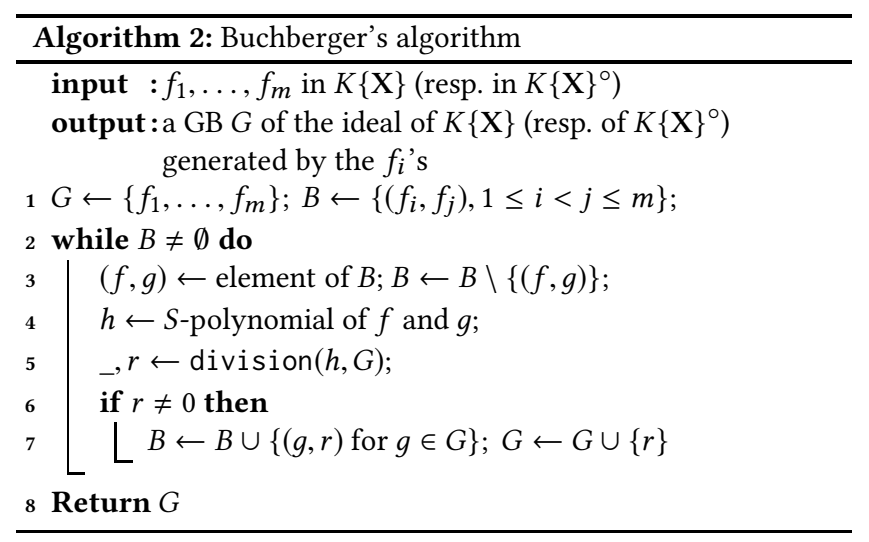

Studying its termination is a bit subtle. Indeed, we have already seen that Algorithm 1 does not terminate in general when we are working at infinite precision. Therefore, Algorithm 2 does not terminate either (since it calls Algorithm 1 on line 5). Nevertheless, one may observe that if, instead of calling Algorithm 1, we ask the reduced form of $h$ modulo $G$ to an oracle that answers instantly, then Algorithm 2 does terminate. In other terms, the only source of possible infinite loops in Algorithm 2 comes from Algorithm 1.

Of course, this point of view is purely theoretical and not satisfying in practice. In practice, the coefficients of $f_{1}, \ldots, f_{m}$ are given at finite precision, i.e. modulo $\pi^{N}$ for some integer $N$, and all the computations are carried out at finite precision. In what follows, we will often use the classical notation $O\left(\pi^{N}\right)$ to refer to a multiple of $\pi^{N}$. In this setting, we have seen that Algorithm 1 does terminate, so Algorithm 2 also terminates. The counterpart is that it is not a priori clear that the result output by Algorithm 2 is a correct approximation of a GB of the ideal we started with. Nevertheless, in the case of $K\{\mathrm{X}\}^{\circ}$, this property holds true as precised by the following theorem.

THEOREM 3.8. Let I be an ideal of $K\{\mathbf{X}\}^{\circ}$ and let $\left(f_{1}, \ldots, f_{m}\right)$ be a generating family of I. Let also $N$ be an integer such that $N>\operatorname{val}(t)$ for all $t \in \operatorname{Skel}(L T(I))$.

When Algorithm 2 is called with $f_{1}+O\left(\pi^{N}\right), \ldots, f_{m}+O\left(\pi^{N}\right)$, it outputs $G=\left(g_{1}, \ldots, g_{s}\right)$ with the following properties:
(1) each $g_{i}$ is known at precision at least $O\left(\pi^{N}\right)$, and

(2) $G$ is the approximation of an actual GB of I.

Proof. The fact that the precision on the $g_{i}$ 's does not decrease follows from the fact that Algorithm 2 only performs "exact" divisions ( $c f$ Remark 3.2).

We now prove (2). Since the $g_{j}$ 's are obtained as linear combinations of the inputs $f_{i}+O\left(\pi^{N}\right)$, there exist $\hat{g}_{1}, \ldots, \hat{g}_{s} \in I$ such that $g_{i}=\hat{g}_{i}+O\left(\pi^{N}\right)$ for all $i$. We set $\hat{G}=\left(\hat{g}_{1}, \ldots, \hat{g}_{s}\right)$; it is enough to prove that $\hat{G}$ is a GB of $I$.

Let $I_{N}=I+\pi^{N} K\{\mathrm{X}\}^{\circ}$ and $\hat{G}_{N}=\left(\hat{g}_{1}, \ldots, \hat{g}_{s}, \pi^{N}\right)$. We claim that $\hat{G}_{N}$ is a GB of $I_{N}$. Since it generates $I_{N}$, it is enough to check Buchberger's criterion. By construction, we know that the reduction of $S\left(\hat{g}_{i}, \hat{g}_{j}\right)$ modulo $\hat{G}$ is a multiple of $\pi^{N}$. Hence $S\left(\hat{g}_{i}, \hat{g}_{j}\right)$ reduces to zero modulo $\hat{G}_{N}$. On the other hand, it follows from the definition of S-polynomials that $S\left(\hat{g}_{i}, \pi^{N}\right)$ is divisible by $\pi^{N}$; hence it also reduces to 0 modulo $\hat{G}_{N}$. The claim is proved.

Let $t \in L T\left(I_{N}\right)$. Then $t=L T\left(f+\pi^{N} h\right)$ for some $f \in I$ and some $h \in K\{\mathrm{X}\}^{\circ}$. If $\operatorname{val}(f)<N$, we have $t=L T(f) \in L T(I)$. Otherwise $t$ is a multiple of $\pi^{N}$. We have then proved that $L T\left(I_{N}\right)$ is the ideal generated by $L T(I)$ and the term $\pi^{N}$. This implies that, if $H$ is a GB of $I$, then $H_{N}=H \cup\left\{\pi^{N}\right\}$ is a GB of $I_{N}$. Moreover by our assumption on $\operatorname{Skel}(L T(I))$, if $H$ is minimal then $H_{N}$ is also.

Choose now a minimal GB $H$ of $I$. From what we have done before and Theorem 2.22, it follows that $L T\left(H_{N}\right) \subset L T\left(\hat{G}_{N}\right)$. Besides, since the $g_{i}$ 's do not vanish at precision $O\left(\pi^{N}\right)$, we have $\operatorname{val}\left(\hat{g}_{i}\right)<N$ for all $i$. Consequently, $L T(H) \subset L T(\hat{G})$. In particular $L T(\hat{G})$ generates $L T(I)$, and so $\hat{G}$ is a GB of $I$.

In the case of $K\{\mathrm{X}\}$, we cannot hope to have similar guarantees. Indeed, if we ask from the GB of the ideal I generated by $f_{1}=$ $X+O\left(\pi^{N}\right)$ and $f_{2}=X+O\left(\pi^{N}\right)$, the answer might be either $(X)$ if $f_{1}=f_{2}=X$, or (1) if $f_{1}=X$ and $f_{2}=X+\pi^{N}$, or many other results. The best we can do is to compute a GB of the sub$K\{\mathrm{X}\}^{\circ}$-module over $K\{\mathrm{X}\}$ generated by the $f_{i}$ 's and answer that the obtained result is likely a GB of $I$. In the example considered above, we will end up with the GB $\left(X+O\left(\pi^{N}\right)\right)$, which is certainly the more natural result we may expect.

General log-radii. We now consider the case of a general $\mathbf{r} \in \mathbb{Q}^{n}$. In this situation, the monoid $T\{\mathbf{X} ; \mathbf{r}\}^{\circ}$ no longer admits gcd's. As a basic example, take $\mathbf{r}=\left(\frac{1}{2}, \frac{1}{2}\right)$ and consider the terms $t_{1}=\pi X_{1}$ and $t_{2}=\pi X_{2}$. Then $\operatorname{val}_{\mathbf{r}}\left(t_{1}\right)=\operatorname{val}_{\mathbf{r}}\left(t_{2}\right)=\frac{1}{2}$. So the valuation of $\operatorname{gcd}\left(t_{1}, t_{2}\right)$ should be $\frac{1}{2}$ as well, implying that $\operatorname{gcd}\left(t_{1}, t_{2}\right)$ should be $\sqrt{\pi}$, which is not an element of $T\{\mathrm{X} ; \mathbf{r}\}^{\circ}$. When we are working over $K\{\mathbf{X} ; \mathbf{r}\}$, this issue does not happen since we can freely multiply by any power of $\pi$. Over $K\{\mathrm{X} ; \mathbf{r}\}$, Algorithm 2 works and is correct (althought we have to be careful with the normalization of gcd's in order to avoid losses of precision as much as possible).

Let us now focus on the case of $K\{\mathrm{X} ; \mathbf{r}\}^{\circ}$ which is more complicated. Let $D$ be a common denominator of the coordinates of $\mathbf{r}$, i.e. $D \cdot \mathbf{r} \in \mathbb{Z}^{n}$. We consider the field extension $L=K[\eta]$ with $\eta^{D}=\pi$. The valuation val extends uniquely to $L$; we have $\operatorname{val}(\eta)=\frac{1}{D}$. We define $L^{\circ}, L\{\mathbf{X}\}$ and $L\{\mathbf{X}\}^{\circ}$ accordingly. Observe that $L^{\circ}=K^{\circ}[\eta]$. If $D \cdot \mathbf{r}=\left(r_{1}, \ldots, r_{n}\right)$, we have $L\{\mathrm{X} ; \mathbf{r}\}=L\{\mathrm{Y}\}$ and $L\{\mathrm{X} ; \mathbf{r}\}^{\circ}=L\{\mathrm{Y}\}^{\circ}$ with $Y_{i}=\eta^{r_{i}} X_{i}$. Moreover the valuation $v^{2} l_{\mathbf{r}}$ over $L\{\mathbf{X} ; \mathbf{r}\}$ (resp. $L\{\mathbf{X} ; \mathbf{r}\}^{\circ}$ ) is transformed into the valuation $\operatorname{val}_{0}$ over $L\{\mathrm{Y}\}$ (resp. 
$\left.L\{\mathrm{Y}\}^{\circ}\right)$. The above identifications show that there is a good notion of gcd's and S-polynomials over $L\{\mathrm{X} ; \mathbf{r}\}$ and $L\{\mathrm{X} ; \mathbf{r}\}^{\circ}$, so that eventually Algorithm 2 runs and computes GB over $L\{X ; \mathbf{r}\}$ and $L\{\mathrm{X} ; \mathbf{r}\}^{\circ}$. Before relating those to GB over $K\{\mathbf{X} ; \mathbf{r}\}$ and $K\{\mathbf{X} ; \mathbf{r}\}^{\circ}$, we need to examine the shape of the GB output by Algorithm 2.

Let $\eta^{\mathbb{N}} K\{\mathbf{X} ; \mathbf{r}\}$ be the subset of $L\{\mathbf{X} ; \mathbf{r}\}$ consisting of elements of the form $\eta^{v} f$ for $v \in \mathbb{N}$ and $f \in K\{\mathbf{X} ; \mathbf{r}\}$. Clearly, $\eta^{\mathbb{N}} K\{\mathbf{X} ; \mathbf{r}\}$ is stable by multiplication. Beyond this, one can check that it exhibits additional stability properties:
The F4 strategy can be then summed up as follows:

(1) Collect all S-pairs sharing the smallest degree for the $1 \mathrm{~cm}$ of their leading terms, and prepare their reduction (Algorithm 3).

(2) Reduce them all together (see Remark 3.11).

(3) Update the GB in construction and list of $S$-pairs according to the result of the previous reduction.

(4) Carry on the previous steps until there is no S-pair remaining. The main algorithm is Algorithm 4, with Algorithm 3 as subroutine.

Proposition 3.9. (1) When Algorithm 1 is called with inputs $f, h_{1}, \ldots, h$

$\eta^{\mathbb{N}} K\{\mathbf{X} ; \mathbf{r}\}$, it outputs $q_{1}, \ldots, q_{m}, r \in \eta^{\mathbb{N}} K\{\mathbf{X} ; \mathbf{r}\}$.

(2) If $f, g \in \eta^{\mathbb{N}} K\{\mathbf{X} ; \mathbf{r}\}$, then $S(f, g) \in \eta^{\mathbb{N}} K\{\mathbf{X} ; \mathbf{r}\}$.

From Proposition 3.9, we deduce immediately that, when Algorithm 2 is called with inputs $f_{i} \in K\{\mathrm{X} ; \mathbf{r}\} \subset L\{\mathrm{X} ; \mathbf{r}\}$, the GB it outputs consists of elements of $\eta^{\mathbb{N}} K\{\mathbf{X} ; \mathbf{r}\}$. The following proposition shows that, after minimizing this GB, we obtain a GB of the ideal of $K\{\mathbf{X} ; \mathbf{r}\}^{\circ}$ we started with.

Proposition 3.10. Let I be an ideal of $K\{\mathrm{X}\}^{\circ}$. Let $G$ be a minimal $G B$ of $I \cdot L\{\mathbf{X} ; \mathbf{r}\}^{\circ}$. We assume $G \subset \eta^{\mathbb{N}} K\{\mathbf{X} ; \mathbf{r}\}$. Then $G \subset K\{\mathbf{X} ; \mathbf{r}\}$ and $G$ is a minimal $G B$ of $I$.

Proof. Write $I_{L}=I \cdot L\{\mathrm{X} ; \mathbf{r}\}^{\circ}$. We claim that:

$$
L T\left(I_{L}\right)=\eta^{\mathbb{N}} L T(I) \quad \text { and } \quad I=I_{L} \cap K\{\mathrm{X} ; \mathbf{r}\} .
$$

The inclusion $\eta^{\mathbb{N}} L T(I) \subset L T\left(I_{L}\right)$ is clear, while the reverse inclusion follows from the fact that any $f \in I_{L}$ can be decomposed as $f=f_{0}+\eta f_{1}+\cdots+\eta^{D-1} f_{D-1}$ with $f_{i} \in K\{\mathbf{X} ; \mathbf{r}\}$ for all $i$. Set $J=I_{L} \cap K\{\mathrm{X} ; \mathbf{r}\}$. From $L T\left(I_{L}\right)=\eta^{\mathbb{N}} L T(I)$, we deduce $L T(I)=L T(J)$. Since moreover $J$ obviously contains $I$, we find $I=J$.

Let $g \in G$. Write $L T(g)=\eta^{v} a \mathrm{X}^{\mathbf{i}}$ with $v \in \mathbb{N}, a \in K^{\times}$and $\mathbf{i} \in \mathbb{N}^{n}$. Since $G$ is a minimal GB of $I_{L}$, we know that $L T(g)$ is minimal in $L T\left(I_{L}\right)$. From Eq. (3), we deduce that $L T(g) \in T\{\mathrm{X} ; \mathbf{r}\}$, that is $\eta^{v} a \in K$. Thus $\eta^{v} \in K$ and $g \in K\{\mathbf{X} ; \mathbf{r}\}$ as claimed. The fact that $G$ is a minimal GB of $I$ follows again from Eq. (3).

To conclude this section, we underline that all computations (i.e. Algorithm 1 and the computation of S-polynomials) can be carried out within $\eta^{\mathbb{N}} K\{\mathbf{X} ; \mathbf{r}\}$, representing an element of this set as a pair $(v, f)$ with $v \in \mathbb{N}$ and $f \in K\{\mathbf{X} ; \mathbf{r}\}$. This strategy avoids constructing and working in the field $L$.

\subsection{F4 algorithm}

In the history of the computation of Gröbner bases, the development of Faugère's F4 algorithm [Fa99] has been a decisive cornerstone towards faster algorithms. In this section, we adjust its strategy to the computation of Gröbner bases over Tate algebras. We restrict ourselves to $\mathbf{r}=0$, keeping in mind that the case of general log-radii can be reached using the techniques discussed at the end of $\S 3.2$.

Roughly, the F4 algorithm is an adaptation of Buchberger's algorithm such that all S-polynomials of a given degree are processed and reduced together in a big matrix of polynomials, along with their reducers. The algorithm carries on the computation until there is no S-polynomials to reduce. Over Tate algebras, as there is no degree, we use instead the degree of the $1 \mathrm{~cm}$ of the leading terms of an $S$-pair.
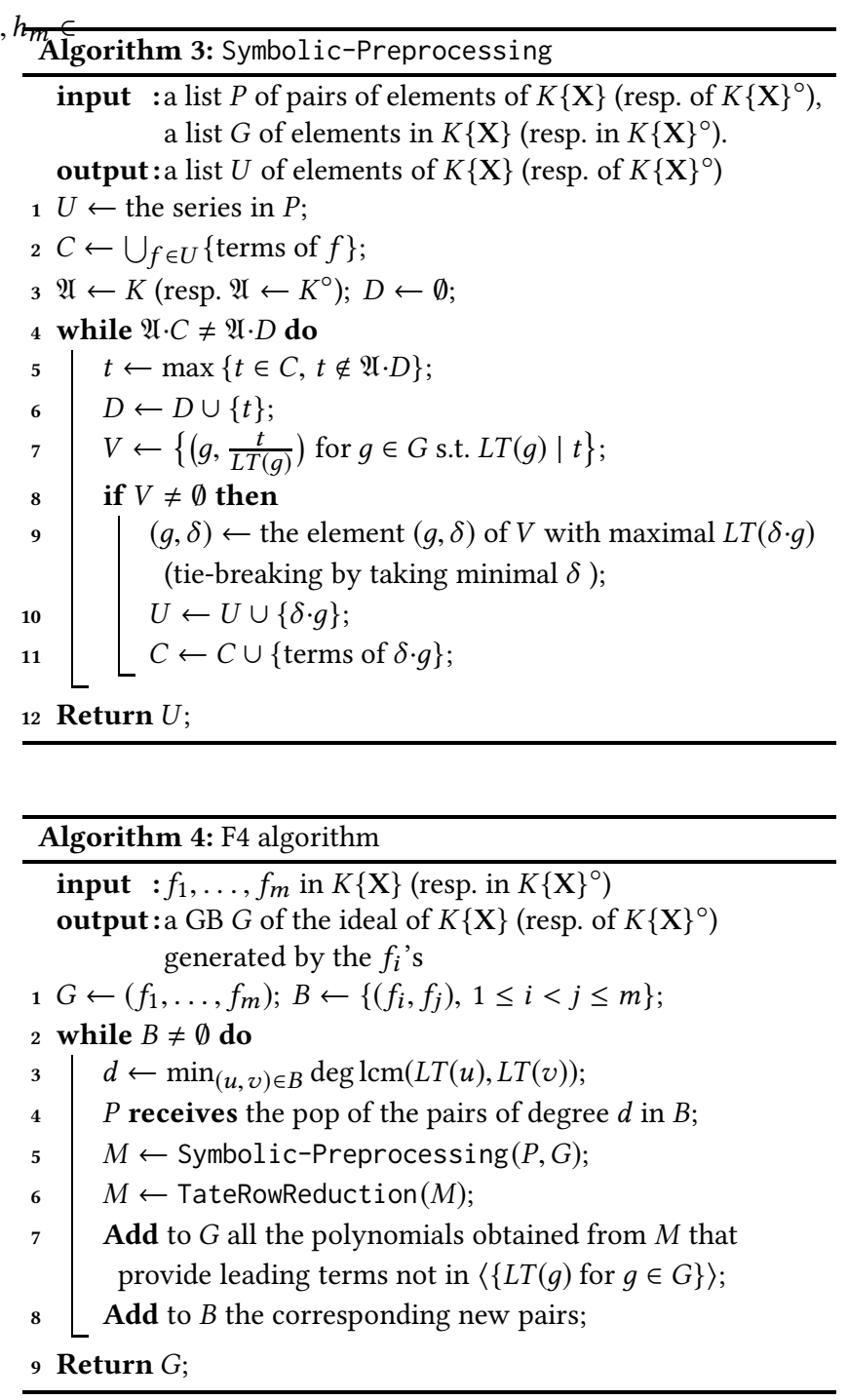

Remark 3.11. On Line 6 of Algorithm 4, the algorithm called TateRowReduction is a simple Smith-Normal-Form-like algorithm: it takes as pivot the not-yet-used series $M_{i}$ with biggest leading term $L T\left(M_{i}\right)$, reduces the other series with this pivot, and proceed until either all remaining series are zeros or all series have served as pivot.

Lemma 3.12. At finite precision, Algorithm 3 terminates in a finite number of steps, and the output $U$ has a finite length. 
Proof. We remark that the sequence formed by the elements $t$ 's considered in the while loop is strictly decreasing. Indeed, we notice first that $t$ is added to $D$ on line 6 , so it cannot reappear later. Then, if $V$ is not empty, all the terms of $\delta \cdot g$ on line 11 are strictly smaller than $t$, except its leading term which is $t$. At finite precision, there is no infinite strictly decreasing sequence by Lemma 2.14 . Consequently, Algorithm 3 terminates in a finite number of steps.

Proposition 3.13. Under the same hypotheses as in Theorem 3.8, Algorithm 4 outputs $G$ satisfying the same conclusions.

Proof. Thanks to Lemma 3.12, it is clear that Algorithm 3 and then the call for TateRowReduction terminate. Termination of Algorithm 4 can then be proved along the following lines. If the algorithm did not terminate for some given input, then it would mean that $B$ (the list of pairs) is never empty. Hence, there would be an infinite number of times when new polynomials are added to $G$. From them, we would be able to construct a strictly increasing sequence of monomial ideals inside $T\{\mathrm{X}\}$ which are nonzero at the precision $O\left(\pi^{N}\right)$. This contradicts Lemma 2.14. Finally, thanks to the Buchberger criterion for Tate algebras ( $c f$ Theorem 3.7), the correctness follows along the same lines as in the proof of Theorem 3.8.

\section{IMPLEMENTATION}

We have implemented in SAGEMATH all the algorithms presented in this paper, together with an interface for working with Tate algebras. Our implementation of Buchberger algorithm ( $c f \S 3.2)$ is now part of the standard distribution of SAGEMATH since version 8.5. It is fairly optimized but it is clear that more work need to be done in this direction: the timings we obtain are far from the average timings reached by other softwares (as SINGULAR) for the computation of Gröbner bases over $\mathbb{Z} / p^{n} \mathbb{Z}$, whereas we could expect them to match, even if the context is a bit different. Our implementation of the $\mathrm{F} 4$ algorithm ( $c f \S 3.3$ ) is still a toy implementation, which does not exhibit good performances yet; we plan to improve it in a near future. It is available at https://gist.github.com/TristanVaccon.

Short demo. Our implementation provides a constructor for creating Tate algebras, called TateAlgebra:

$$
\begin{array}{ll}
\text { In: } & \mathrm{K}=\mathrm{Qp}(2, \text { prec }=5 \text {, print_mode='digits' }) \\
& \mathrm{A} .<x, y>=\text { TateAlgebra(K); } \mathrm{A} \\
\text { Out: } & \mathbb{Q}_{2}\{x, y\}
\end{array}
$$

We observe that, by default, the log-radii are all zero; the keyword log_radii can be use to pass in other values. Similarly the default order is the one attached to $\omega=$ grevlex, but any other order known by SAGEMATH can be specified via the keyword order.

The ring of integers of the Tate algebras can be built as follows:

$$
\begin{aligned}
\text { In: } & \text { Ao }=\text { A.integer_ring(); Ao } \\
\text { Out: } & \mathbb{Q}_{2}\{x, y\}^{\circ}
\end{aligned}
$$

We can now create and manipulate elements:

$$
\begin{aligned}
\text { In: } & f=2 * x^{\wedge} 2+5 * x * y^{\wedge} 2 \\
& g=4+2 * x^{\wedge} 2 * y \\
& f+g \\
\text { Out: } & \ldots 00101 x y^{2}+\ldots 000010 x^{2} y+\ldots 000010 x^{2}+\ldots 0000100 \\
\text { In: } & (1+g) . \text { inverse_of_unit }() \\
\text { Out: } & \ldots 01101+\ldots 01110 x^{2} y+\ldots 10100 x^{4} y^{2}+ \\
& \ldots 11000 x^{6} y^{3}+\ldots 10000 x^{8} y^{4}+O\left(2^{5} \mathbb{Q}_{2}\{x, y\}^{\circ}\right)
\end{aligned}
$$

We observe that, in the outputs, terms are ordered with respect to the term order on $T\{\mathbf{X}\}$, the greatest one coming first. The big-oh appearing on the last line hides terms which are multiple of $2^{5}$.

Classical transcendantal functions are also implemented, e.g.:

$$
\begin{aligned}
\text { In: } & \log (1+\mathrm{g}) \\
\text { Out: } & \ldots 01110 x^{4} y^{2}+\ldots 11010 x^{2} y+\ldots 11100 x^{8} y^{4}+ \\
& \ldots 11100+\ldots 11000 x^{6} y^{3}+O\left(2^{5} \mathbb{Q}_{2}\{x, y\}^{\circ}\right)
\end{aligned}
$$

Ideals of $K\{\mathrm{X}\}$ can be defined and manipulated as follows:

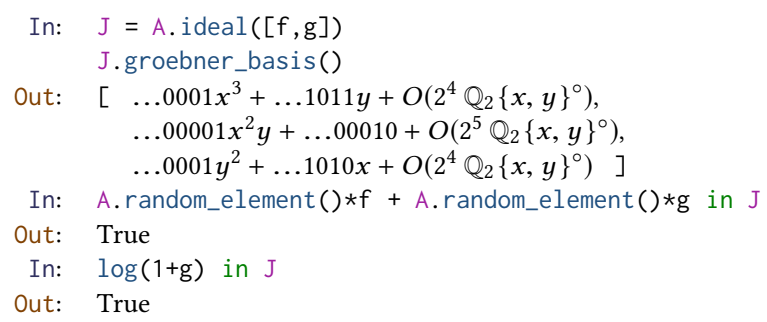

And similarly for ideals of $K\{\mathbf{X}\}^{\circ}$ (observe that no losses of precision occur this time, in accordance with Theorem 3.8):

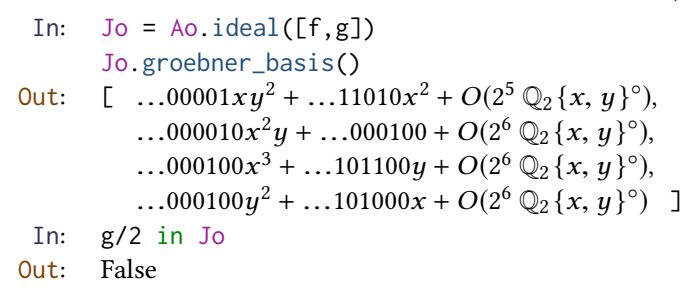

\section{REFERENCES}

[AL94] Adams William and Loustaunau Philippe, An Introduction to Gröbner Bases, Amer. Math. Soc. 7 (1994)

[BGR84] Bosch Siegfried, Günzter Ulrich and Remmert Reinhold, Non-Archimedean analysis, Springer-Verlag (1984)

[Bu65] Buchberger Bruno, Ein Algorithmus zum Auffinden der Basiselemente des Restklassenringes nach einem nulldimensionalen Polynomideal (An Algorithm for Finding the Basis Elements in the Residue Class Ring Modulo a Zero Dimensional Polynomial Ideal), English translation in J. of Symbolic Computation, Special Issue on Logic, Mathematics, and Computer Science: Interactions. Vol. 41, Number 3-4, Pages 475-511, 2006

[CL14] Caruso Xavier and Lubicz David, Linear Algebra over $\mathbb{Z}_{p}[[u]]$ and related rings, LMS J. Comput. Math. 17 (2014), 302-344

[CM19] Chan Andrew and Maclagan Diane, Gröbner bases over fields with valuations, Math. Comp. 88 (2019), 467-483.

[Co15] David A. Cox, John Little, and Donal O'Shea. Ideals, varieties, and algorithms. Undergraduate Texts in Mathematics. Springer, Cham, fourth edition, 2015.

[Fa99] Faugère Jean-Charles, A new efficient algorithm for computing Gröbner bases (F4), Journal of Pure and Applied Algebra, 1999

[GR95] Gräbe Hans-Gert, Algorithms in Local Algebra, Journal of Symbolic Computation 19, 1995, 545-557

[KC09] Kapur Deepak and Cai Yongyang, An Algorithm for Computing a Gröbner Basis of a Polynomial Ideal over a Ring with Zero Divisors, Mathematics in Computer Science, 2009

[Mo82] Mora Ferdinando, An algorithm to compute the equations of tangent cones, Proceedings of European Computer Algebra Conference in Marseille, 1982, 158165

[Sage] SageMath, the Sage Mathematics Software System (Version 8.6), The Sage Development Team, 2018, http://www.sagemath.org

[NS01] Graham H. Norton and Ana Sălăgean, Strong Grobner bases and cyclic codes over a finite-chain ring. Electronic notes in discrete maths 6, 2001, 240-250

[Ta71] Tate John, Rigid analytic spaces, Inventiones Mathematicae 12, 1971, 257-289 
[Va14] Vaccon Tristan, Matrix-F5 algorithms over finite-precision complete discrete valuation fields, Proceedings of 39th International Symposium on Symbolic and Algebraic Computation, ISSAC'14, Kobe, Japan.

[Va $\left.{ }^{*}\right]$ Vaccon Tristan, Précision $p$-adique, thèse de l'Université de Rennes 1 , https://tel.archives-ouvertes.fr/tel-01205269.

[Va15] Vaccon Tristan, Matrix-F5 Algorithms and Tropical Gröbner Bases Computation, Proceedings of the 40th International Symposium on Symbolic and Algebraic Computation, ISSAC 2015, Bath, United Kingdom. Extended version in the Journal of Symbolic Computation, Dec. 2017.

[VY17] Vaccon Tristan and Yokoyama Kazuhiro, A Tropical F5 algorithm, Proceedings of the 42th International Symposium on Symbolic and Algebraic Computation, ISSAC 2017, Kaiserslautern, Germany.

[VVY18] Vaccon Tristan, Verron Thibaut and Yokoyama Kazuhiro, On Affine Tropical F5 algorithm, Proceedings of the 43th International Symposium on Symbolic and Algebraic Computation, ISSAC 2018, New York, USA 